\title{
Series-Resonant Single-Phase AC-DC Power Supply with Control of Reactive Power
}

\author{
J. Ben Klaassens, Marinus P. N. van Wesenbeeck, and Hian K. Lauw
}

\begin{abstract}
The series-resonant converter is one of the major classes of electronic power converters that are extensively applied in dc-dc conversion. This technology transfers bulk energy efficiently through a series-resonant circuit in the direct path of the energy transfer. This resonant converter forms $a$ high-frequency ac link between the voltage sources connected to the input and output terminals. The high-frequency link is exploited in the application of a modulation process, thus avoiding the use of bulky and expensive low-order harmonic filters. The achievable high-pulse repetition frequency is rooted in the efficient turn-off mechanism of the semiconductor switches and does not compromise their reliability.

The classical objective of an ac-dc converter is to control the (average value of the) output waveform (often the output voltage). The energy emanated from the source is adapted by passive filters, often a compromise between volume and effective use. A rectifier-filter network presents a major disadvantage: a power factor less than one caused by distortion of the source current.

The resolution of the high-frequency input current of the series-resonant converter introduces the possibility of accurately controlling the waveform of the source current. In combination with the accurate positioning of the high-frequency current pulses, it is possible to influence the exchange of power with the source by the introduced method of active filtering. A power factor equal to one would lead to optimal operation.

However the zero crossing of the ac source voltage introduces the necessity to store energy. Bulky low-frequency storage elements increase the specific volume and weight of the converter, while the exchange of energy with storage elements decrease the overall efficiency. The energy stored in the resonant circuit is fundamentally too low to solve this problem. The stored energy in the output capacitor necessary to decrease the highfrequency ripple voltage is available for these purposes. To meet the optimal conditions for the power factor at the source, a bipolar flow of energy is required.
\end{abstract}

\section{Power Circuit Configuration}

$\mathrm{E}$ $\mathrm{ACH}$ input and output line is connected to the switching elements. The configuration in Fig. 1 applies to a single-phase input and output converter. As shown in this figure the sinusoidal source voltage $e_{s}$ is connected to the switching matrix SM1, whereas the load with a dc voltage $u_{o}$ is connected to the switching matrix SM2. Both switching matrices are of the half-bridge configuration. The switching matrices are interconnected by a series-resonant circuit constructed with the passive components $L_{r}$ and

Manuscript received February 5, 1990; revised April 26, 1991

J. B. Klaassens and M. P. N. van Wesenbeeck are with Delft University of Technology, $2600 \mathrm{GA}$ Delft, The Netherlands.

H. L. Lauw is with Oregon State University, Corvallis, OR 97222. IEEE Log Number 9105011
$C_{r}$. It is further to be noted that each switching element is realized by two semiconductors in antiparallel in order to be able to switch the bipolar resonant current $i_{r}$ for each polarity of the voltages connected to the board. This topology facilitates dc and ac input and output and provides the capability of bidirectional power flow, i.e., fourquadrant operation.

The resonant circuit elements of the proposed converter in Fig. 1 are formed by the inductor $L_{r}$ and the parallel combination of the capacitors $C_{r 1}=\frac{1}{2} C_{r}$ and $C_{r 2}=\frac{1}{2} C_{r}$. Capacitors $C_{s}$ and the combination of $C_{o 1}=\frac{1}{2} C_{o}$ and $C_{o 2}$ $=\frac{1}{2} C_{o}$ are the input and output filter capacitors that are inserted to form a low-impedance path for the high-frequency components of the source and output currents, respectively. Capacitors $C_{s}$ and $C_{o}$ should be large compared to the resonant capacitor $C_{r}$.

This power circuit does not need additional components in order to ensure

1) Natural commutation

2) Unity power factor at the input (no power factor boost circuit is required)

3) An internal frequency as high as necessary by the desire to meet the requirements with respect to the output performance specification, size, and power density without the need to include bulky and expensive low-order harmonic filters

4) Operation of the converter with ac and dc input

5) Bidirectional power flow and four-quadrant operation

The use of thyristors makes it possible to build a seriesresonant converter with an internal converter frequency of up to a hundred kilohertz limited by the turn-off time of commercially available thyristors. The use of power FET's makes it possible to increase this frequency to beyond $500 \mathrm{kHz}$. The use of staggered power modules increases the pulse repetition frequency at the output port of the converter without increasing the internal frequency and, consequently, improves the indicated mode of modulation [10]. It is noted that size advantage with increasing frequency is not present in this class of power converters, which has to apply a dc link. The thyristor is significantly cheaper and more robust than gate turn-off switches (GTO's) in addition to facilitating convenient interfacing between the control circuit and the power circuit due to the low gate current required. 


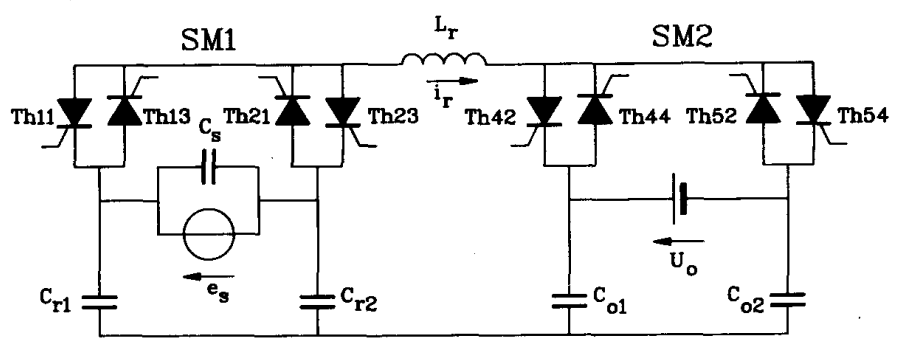

Fig. 1. Diagram power circuit of the series-resonant single-phase ac-dc converter.

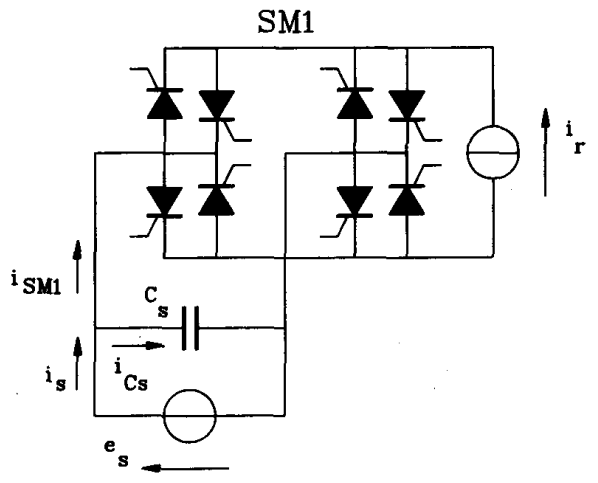

(a)
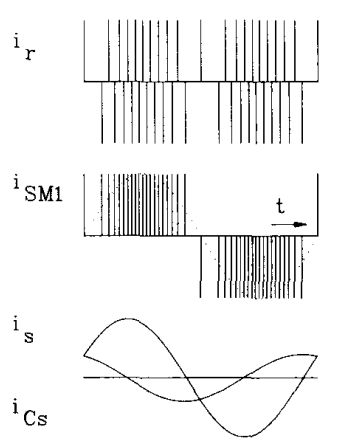

(b)

Fig. 2. Process of pulse positioning and modulation. (a) Equivalent circuit diagram. (b) Characteristic waveforms.

Fig. 2 presents the process of pulse positioning and modulation of the source-sided current pulses $i_{\mathrm{SM} 1}$ on the switching matrix SM1. The high-frequency carrier $i_{r}$ as generated by the series-resonant converter is modulated in frequency and in combination with a low-frequency, bipolar programmed process of rectification; a sinusoidal source current $i_{\text {SM }}$ is generated at the input of the switching matrix SM1. The fundamental component of this current (as depicted in Fig. 2(b) by the dotted line), in combination with the capacitor current $i_{C s}$, has to be positioned in phase with the sinusoidal source voltage $e_{s}$. The harmonic components of the pulse modulation process have only a minor influence on the power factor. The current $i_{\mathrm{SM} 1}$ is generated independently of the source voltage $e_{s}$. It is necessary to operate the source-sided switching matrix SM1 as an inverter-type rectifier energized by an en- ergy source different from the voltage source $e_{s}$. Active compensation of the power factor of a system is only feasible for four-quadrant operation. Two-quadrant operation (exchange of power with the source in one direction) will result in a degradation of the power factor, especially at low-load conditions.

\section{Conversion Process}

The exchange of energy is activated by the combination of the two voltage sources $e_{s}$ and $u_{o}$ by selecting a proper combination of switches. Switching matrices SM1 and SM2 excite the series-resonant circuit by the voltage $u_{L C}$ as a combination of the $\mathrm{AC}$ voltages $e_{\mathrm{SM} 1}$ and $e_{\mathrm{SM} 2}$ :

$$
u_{L C}=e_{\mathrm{SM} 1}-e_{\mathrm{SM} 2}
$$

where $e_{\mathrm{SM} 1}=\frac{1}{2} e_{s}$ and $e_{S M 2}=\frac{1}{2} u_{o}$ as usual for a half-bridge switching matrix. It is sufficient to introduce two voltage sources $\left|e_{\mathrm{SM} 1}\right|=\frac{1}{2} e_{s}$ and $\left|e_{\mathrm{SM} 2}\right|=\frac{1}{2} u_{o}$. The converter network is now replaced by the equivalent network shown in Fig. 3. Both voltage sources represent the selected sources at the outside of the converter for a single resonant current pulse. The operation of the controlled series-resonant circuit is based on the excitation by the resulting voltage $u_{L C}$ resulting in a modulated high-frequency carrier $i_{r}$.

The resonant current $i_{r}$ will pass a selection of both voltage sources as connected to the switches. Depending on the configurations of the active switches in the matrix $S M p$ an ac voltage $e_{S M p}$ is supplied at the terminals of the matrix or by selecting the voltage source or by bypassing the voltage source. The ac voltage $e_{S M p}(p=1,2)$ is selected from the two possible values $+e_{s}$ or $-e_{s}$ and $+u_{0}$ or $-u_{o}$. For the half-bridge configuration as presented in Fig. 1 , the resulting excitation voltage $u_{L C}(1)$ is limited to four combinations of $\frac{1}{2} e_{s}$ and $\frac{1}{2} u_{o}$ [9]:

$$
\pm \frac{1}{2}\left(e_{s}+u_{o}\right), \pm \frac{1}{2}\left(e_{s}-u_{o}\right) \text {. }
$$

The result is a sinusoidal resonant current $i_{r}$ and resonant capacitor voltage $u_{C r}$ with low damping. The multiplication of the resonant current $i_{r}$ and the excitation voltage $u_{L C}$ describes the exchange of energy with the resonant circuit.

The control of the semiconductor switches in both matrices has to guarantee two conditions:

1) A net amount of energy has to be distributed in the desired direction for each individual current pulse. 


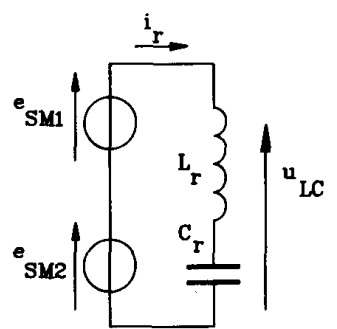

Fig. 3. Double excited resonant circuit.

2) A recurrent change of stored energy in the network has to be avoided.

Electric energy is distributed from a source $e_{s}$ to a source $u_{o}$ by a proper selection of sources: $\left(e_{s}-u_{o}\right) i_{r}>$ 0 . The difference of the energy emanated from the source and the energy distributed to the load will change the energy level of the resonant circuit. For $e_{s}>u_{o}$ the converter system can be regarded as a step-down converter for which the stored energy in the resonant capacitor will recurrently increase. For $e_{s}<u_{o}$ the converter system can be regarded as a step-up converter for which the stored energy in the resonant capacitor will recurrently decrease. To stabilize the amplitude of the resonant capacitor voltage $u_{C r \max }$ at the end of each individual current pulse in order to obtain a cyclic stable mode of operation, the energy distribution to the resonant circuit has to be controlled during each current pulse. A resonant current pulse is hence build up from two segments (i.e., two levels for the excitation voltage). One current segment is applied to distribute power. The other current segment will stabilize the stored energy by a process of active damping in contrast to passive damping, which will increase the circuit losses. The energy level of the $L C$ circuit has to be increased by selecting an additional excitation voltage $u_{L C}$ : $u_{L C} i_{r}>0$ or decreased by selecting an additional excitation voltage $u_{L C}: u_{L C} i_{r}<0$.

The characteristic waveforms of the resonant circuit are shown in Fig. 4(a) for the step-down mode. The energy is exchanged during the time interval indicated as $\psi_{f k}$ where the excitation voltage $u_{L C}$ is the difference of both voltage sources $e_{s}$ and $u_{o}$. The excess of energy during the time interval $\psi_{f k}$ is returned to both external voltage sources during the time interval $\psi_{r k}$. To satisfy the turnoff conditions of the thyristor switches time intervals $\psi_{r k}$ are preceding $\psi_{f k}$. A variation of the phase angle $\psi_{r k}$ provides a change in the maximal capacitor voltage $u_{C r m a x}$. The characteristic waveforms of the resonant circuit are shown in Fig. 4(b) for the step-up mode. The energy is exchanged during the time interval indicated as $\psi_{r k}$ where the excitation voltage $u_{L C}$ is the difference between both voltage sources $e_{s}$ and $u_{o}$. For this mode it is necessary to supply additional energy from both voltage sources $e_{s}$ and $u_{o}$ to the resonant network to prevent the interrupt of the distribution of power from the source to the load. Therefore, a boost phase with a time length $\psi_{f k}$ is introduced with an excitation voltage $u_{L C}$ equal to the sum of both

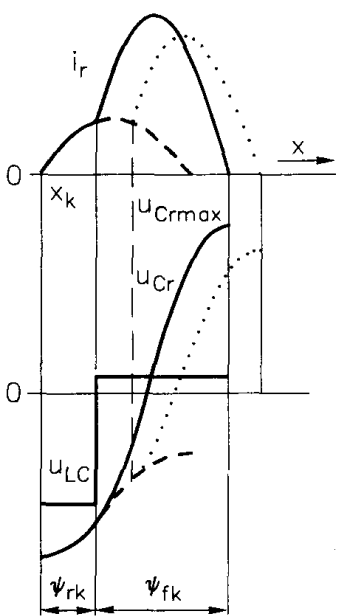

(a)

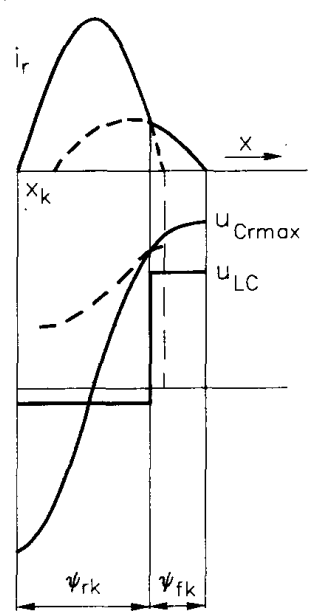

(b)
Fig. 4. Resonant inductor current $i_{r}$, resonant capacitor voltage $u_{C r}$, and * excitation voltage $u_{L C}$ for (a) step-down mode, (b) step-up mode

external voltages. The indicated phases $\psi_{r k}$ and $\psi_{f k}$ are repeated sequentially with alternating polarity. The control of the time intervals $\psi_{r k}$ and $\psi_{f k}$ of each current segment is accomplished for the exchange of energy with the resonant circuit and the distribution of power between the source and the load or vice versa over one half cycle. The direction for the exchange of energy between the source $e_{s}$, the load with a voltage $u_{o}$, and the resonant circuit is selected by the appropriate switches in both matrices. The equal rank of all matrices in the conversion network allows the direction of the power flow to be mutually exchanged. It is possible to exchange electric energy between both sources in two directions.

Each conversion process is implemented by the selection of at least one switching matrix connected to the voltage source with the lowest voltage level $e_{l}$ operating as a common rectifier synchronized at the zero crossings of the resonant current and one switching matrix operating as a rectifier connected to the voltage source with the highest voltage level $e_{h}>e_{l}$ synchronized to the resonant current but shifted in phase with respect to the zero crossings of the resonant current to guarantee the introduced process of active damping by the phase control of the switching instants. A bipolar switching matrix current $i_{h}$ is exchanged with this voltage source $e_{h}$ in order to stabilize the stored energy (and consequently the amplitudes of the critical waveforms) of the resonant circuit.

Selecting the correct combination of the excitation voltages opens the way to the exchange of electric energy between two voltage sources interconnected by a resonant circuit for any ratio of the voltages $e_{s}$ and $u_{o}$ (even for $e_{s}$ $\approx u_{o}$ ). In reality, a converter shows electric losses that are rooted in the voltage drop of the semiconductor switches and the conduction losses of the passive components and wiring. The transfer of energy will be interrupted for a series-resonant conversion system if its conversion ratio $q \approx 1$. For this mode of operation; electric 


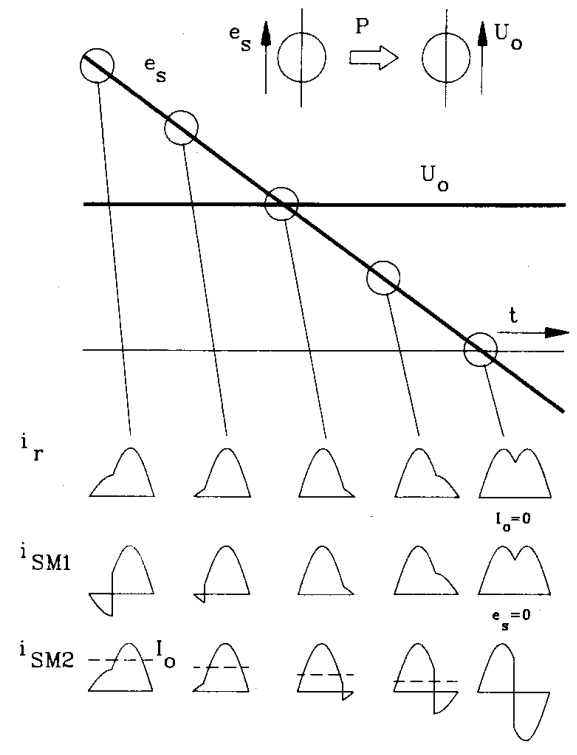

Fig. 5. Waveforms generated by the resonant circuit for different values of the source voltage $e_{s}$ and output voltage $u_{o}$. $i_{r}$ : resonant current. $i_{\mathrm{SM} 2}$ : unfiltered output current. $i_{\mathrm{SM} 1}$ : unfiltered source current.

energy is distributed for an effective excitation voltage $u_{L C}=0$ indicating that no energy is added to the resonant circuit. Ohmic losses will finally decrease the amplitude of the resonant capacitor voltage until the oscillation is interrupted. For each current pulse the losses have to be compensated to maintain the energy level of the switched resonant circuit. It is required to transfer energy to the resonant circuit. The configuration of the switches is modified in order to generate the necessary excitation voltage $u_{L C}$ for which $u_{L C} i_{r}>0$ in order to boost the stored energy in the resonant circuit as discussed with reference to Fig. 4(b).

Fig. 5 illustrates the resonant current $i_{r}$ and the unfiltered source and output current $i_{\mathrm{SM} 1}$ and $i_{\mathrm{SM} 2}$ characterizing the described processes in the double excited seriesresonant circuit. The source voltage $e_{s}$ is a time-varying (sinusoidal) waveform, whereas the output voltage $u_{o}$ is a constant dc voltage. The current segments indicating the return of energy from or the supply of energy to the resonant circuit are well indicated.

\section{Energy Storage}

A sinusoidal voltage source $e_{s}=\hat{e}_{s} \sin \left(\omega_{s} t\right)$ is connected to the switching matrix SM1. The phase shift between the source voltage $e_{s}$ and the fundamental source current $i_{s}$ is presented by the phase angle $\varphi_{s}$. The source current $i_{s}$ is constructed from two components $i_{s q}$ and $i_{s d}$ equal to the orthogonal functions.

$$
i_{s}=i_{s q}+i_{s d}=\hat{\imath}_{s} \sin \left(\omega_{s} t+\varphi_{s}\right)
$$

where

$$
\begin{aligned}
& i_{s d}=\hat{i}_{s d} \sin \left(\omega_{s} t\right) \\
& i_{s q}=\hat{i}_{s q} \cos \left(\omega_{s} t\right) .
\end{aligned}
$$

The momentary value of the power as emanated from the source by the converter is

$$
\begin{aligned}
S_{s} & =P_{s}+Q_{s}=e_{s} i_{s} \\
& =\frac{1}{2} \hat{e}_{s} \hat{\imath}_{s}\left[\cos \left(\varphi_{s}\right)-\cos \left(2 \omega_{s} t-\varphi_{s}\right)\right]
\end{aligned}
$$

where $P_{s}$ is constant. To obtain a time-independent flow of power, the time-varying reactive power $Q_{s}$ has to be generated by a separate energy buffer. To avoid additional circuitry the output capacitor $C_{o}$ is available to fulfill this function next to its function as ripple suppressor. Energy can now be returned to the source voltage with very low values. This explains the four-quadrant operation.

A constant flow of power at the output terminals is achieved if an energy buffer is added to the conversion system. The amount of power absorbed by the dc load is nearly constant in magnitude and equals the real input power $P_{s}$. The output capacitor absorbs the total amount of

$$
Q_{C o}(t)=\frac{1}{2} \hat{e}_{s} \hat{l}_{s} \cos \left(2 \omega_{s} t-\varphi_{s}^{u}\right)
$$

where the phase angle $\varphi_{s}^{u}$ is introduced as the phase angle for an unregulated source current, i.e., the fundamental component of the matrix current $i_{\mathrm{SM} 1}$ (see Fig. 2(b)) is in phase with its source voltage $e_{s}$. The optimal capacitor size $C_{o \text { min }}$ is given by the relation

$$
\mathrm{C}_{o \min }=\frac{P_{o}}{\omega_{s} U_{o} \Delta u_{o} \cos \left(\varphi_{s}^{u}\right)} .
$$

For this condition the power factor is equal to one. Equation (9) shows that the output voltage $U_{o}$ has to be unequal to zero as is expected. The output capacitor $C_{o}$ as found in resonant $\mathrm{dc}-\mathrm{dc}$ converters without reactive power control is about seven times smaller in value for the same peak-to-peak output ripple voltage $\Delta u_{o}$.

\section{CONTROL}

The shape of the source current $i_{s}$ is controlled by a pulse modulation process as presented in Fig. 2 and has to be sinusoidal. The control has to minimize the quadrature component $i_{s q}$ of the source current resulting in a power factor equal to one. The amount of distributed power is controlled by the direct component $i_{s d}$ of the source current. The block diagram of the control system is shown in Fig. 6. In the control system two control loops are distinguished:

1) The low-frequency outer loop governs the (average) dc output voltage and produces a reference current 


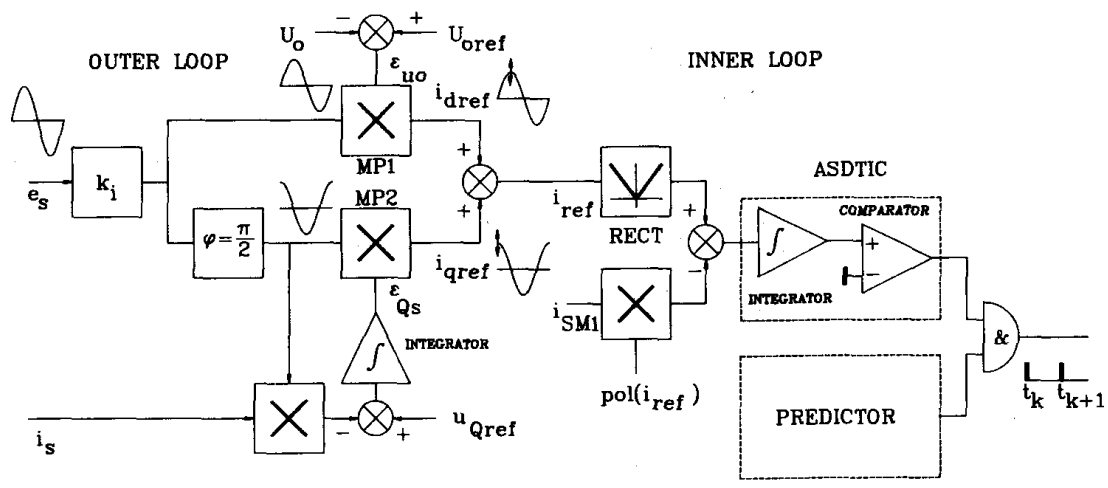

Fig. 6. Block diagram of the control system.

$i_{\text {ref }}$ for the inner-loop ASDTIC controller (ASDTIC: analog signal to discrete time interval converter [2]).

2) The high-frequency inner loop controls the pulse modulation process of the converter. An ASDTICpulse modulation process enables the generation of the low-frequency input current $i_{s}$ with low distortion.

Four control functions are identified and implemented in both control loops: 1) control source current, 2) control output voltage, 3) predictor control, and 4) pulse modulator. The outer loop independently controls the $d$ and $q$ component of the source current $i_{s}$. The reference signal $i_{d \text { ref }}$ and $i_{\text {qref }}$ are directly derived from the sinusoidal source voltage $e_{s}$. The amplitudes of both current components $i_{s d}$ and $i_{s q}$ can be controlled independently by the dc reference signals $U_{\text {oref }}$ and $U_{\text {Qref }}$.

The amplitude of $i_{d r e f}$ is controlled by an error signal $\epsilon_{u o}=U_{o r e f}-U_{o}$. The amplitude of this reference signal is controlled by a multiplier MP1. This value is the error signal of the control loop for the output voltage $U_{o}$ with its reference signal $U_{\text {oref }}$. The inevitable internal converter losses are compensated automatically. The amplitude of $i_{\text {qref }}$ is under the control of the error signal $\epsilon_{Q s}=i_{s}-u_{Q \text { ref }}$ by multiplier MP2. For a power factor of one, reference voltage $u_{Q \text { ref }}$ has to be set to zero. Reference signal $i_{\text {ref }}$ is composed of $i_{\text {refq }}$ and $i_{\text {ref } d}$ (following (3)). The current $i_{\text {SM1 }}$ is multiplied by the polarity pol $\left(i_{\text {ref }}\right)$ for which $\operatorname{pol}\left(i_{\text {ref }}\right)=$ +1 or -1 for $e_{s} \geq 0$ or $e_{s}<0$, respectively. The pulseposition control is realized by an ASDTIC controller with its input signals $i_{\text {ref }}$ and the rectified switching current $\left|i_{\text {SM } 1}\right|$ where

$$
\int_{t_{k}}^{t_{k+1}}\left[\left|i_{\mathrm{SM} 1}\right|-\left|i_{\mathrm{ref}}\right|\right] d t=0 .
$$

The charge of each individual current pulse over each time interval $\left[t_{k}, t_{\mathrm{k}+1}\right]$ is equal to the average value of the reference signal $\left|i_{\text {ref }}\right|$ over the same interval. The thyristor switches are fired by the output signal of the comparator at the time $t_{k}(k=1,2,3, \cdots)$. An uninterrupted flow of power can be guaranteed by using a dual-predictor control algorithm [9]. The predictor circuit will maintain a constant level of the maximal energy in the resonant capacitor (i.e., amplitudes of the resonant capacitor voltage) for each individual current pulse in a predictive way.

\section{Results of the Simulation}

The results of a computer simulation of a series-resonant converter including losses confirm the indicated phenomena. The operation of the introduced method is illustrated in Fig. 7. The controlled firing process of the semiconductor switches in matrix SM1 will generate a current $i_{\mathrm{SM} 1}$ as indicated in this figure. The individual pulses are derived by a modulated process of rectification from the high-frequency carrier $i_{r}$. The source current $i_{s}$ is the result of the current through the input filter capacitor $C_{s}$ and matrix current $i_{\mathrm{SM} 1}$. In Fig. 7(a) the source current $i_{s}$ is controlled for a source-sided phase shift $\varphi_{s}=0$ whereas in (b) this current is presented for $\varphi_{s}=\pi / 4$ (leading current). In Fig. 7(a) the value for the reference signal is $i_{\text {gref }} \neq 0$, whereas in Fig. $7(b)$ it is obvious that the same reference signal is now zero (unregulated mode of operation).

\section{Results of ExPERIMENTS}

An experimental series-resonant converter will be tested with respect to functional integrity under adverse conditions of operation. The conversion system applying thyristor switches, generating a constant dc output voltage at the output terminals, has been designed and constructed to exchange $1 \mathrm{~kW}$ of power with an sinusoidal ac source with a maximum voltage $\hat{e}_{s}=311 \mathrm{~V}\left(e_{s r m s}=220 \mathrm{~V}\right)$ and a constant frequency $f_{s}=50 \mathrm{~Hz}$. The dc output voltage $U_{o}$ has a value of $300 \mathrm{~V}$. The output power is $1 \mathrm{~kW}$ and is the result of a pulsating flow of energy but with varying pulse frequency. The energy stored in the output capacitor 


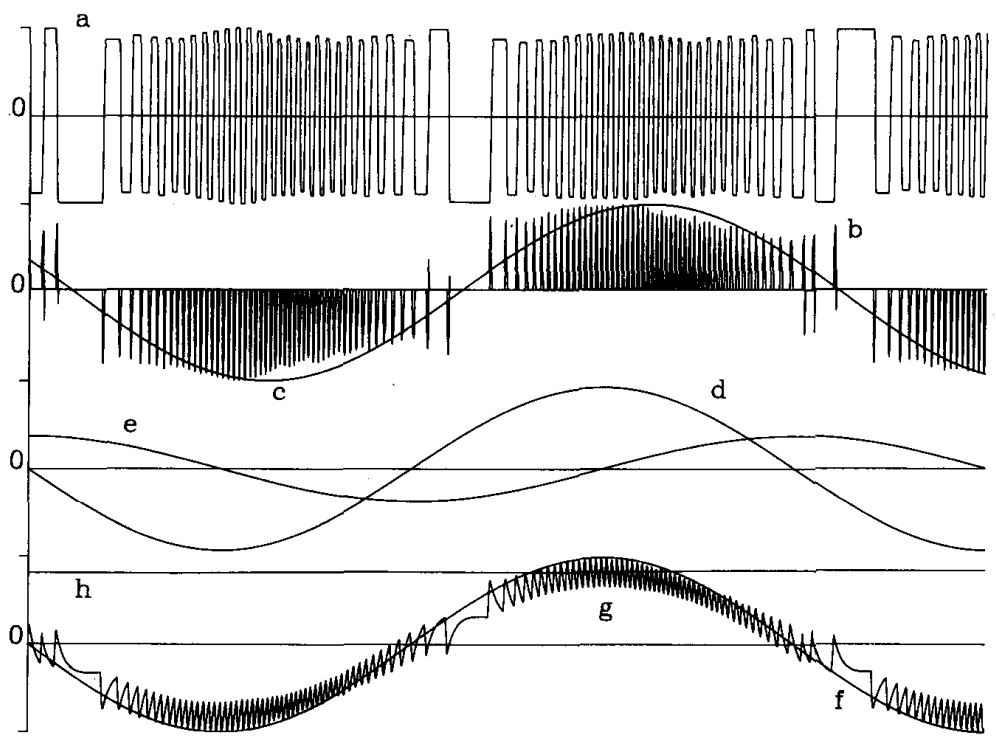

(a)

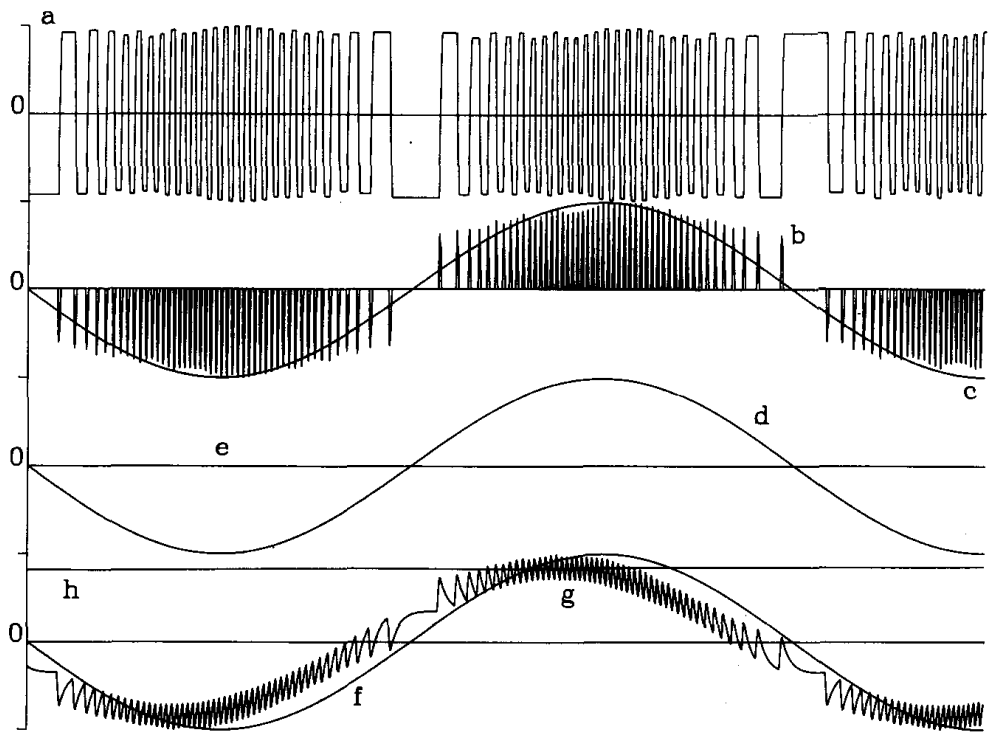

(b)

Fig. 7. Results of simulation for the following waveforms. (a) Capacitor voltage $u_{C r}$. (b) Input current switching matrix $i_{\mathrm{SM}}$. (c) Reference signal $i_{\text {ref. }}$. (d) Reference signals $i_{d \text { ref }}$. (e) Reference signals $i_{q \text { ref. }}$. (f) Source voltage $e_{s}$. (g) Source current $i_{s}$. (h) DC output voltage $u_{o}$. For two values of the source-sided phase shift $\varphi_{s}$. (a) $\varphi_{s}=0$. (b) $\varphi_{s}=\pi / 4$ (leading current).

is also used to compensate the reactive power. Therefore the resonant converter is designed for a peak power of about twice the average output power. The peak device voltage is 2.2 times $\hat{e}_{s}$, whereas the peak device current is 6.5 times the dc output current. The maximal stored energy in the passive resonant components are equal to $\epsilon_{C r}=0.07 \mathrm{~J}$ and $\epsilon_{L r}=0.07 \mathrm{~J}$. The characteristic waveforms of the source voltage and current confirm the char- acteristics of the power conversion process to have a linear resistive input impedance and are presented in Fig. 8 for a power factor of one or one-half. These waveforms are derived from a similar converter system and confirm the presented method and can be compared with the results of simulation in Fig. 7. For all conditions of operation the amplitude of the resonant capacitor voltage is kept constant by the dual-predictor controller. 


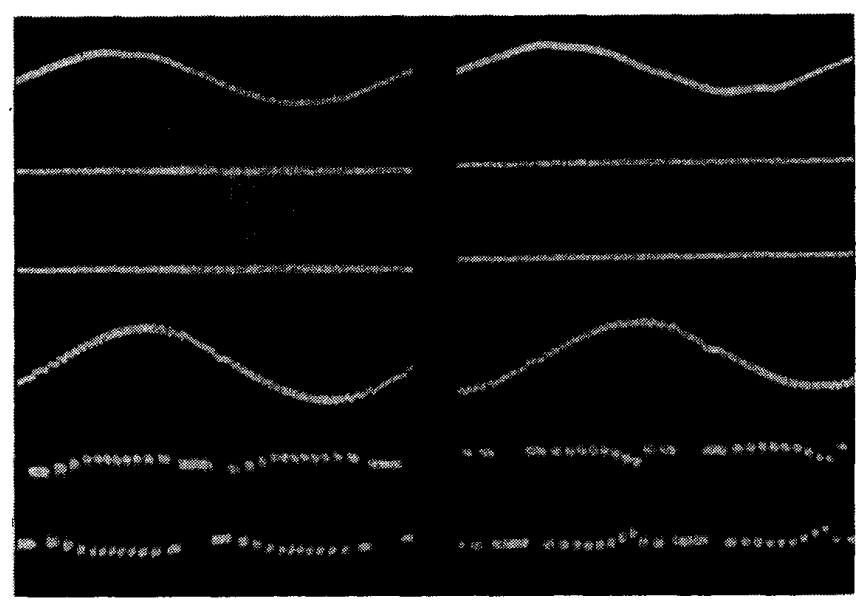

Fig. 8. Experimental waveforms for (a) phase angle $\varphi_{s} \approx 0$, (b) phase angle $\varphi_{s}=\pi / 3$ (lagging).

\section{Conclusions}

The classical rectifier-filter network, as usually applied to ac-dc converters, limits the available grid power due to the degradation of the power factor [1]. An harmonic free interface achieves a power factor of one and permits a substantial increase in the power.

Different schemes for switching networks with active power factor compensation including dc operation, were proposed [3]. The presented method of active filtering is implemented by a conversion system with thyristor switches generating a high-frequency carrier for the distribution of energy.

To avoid additional low-frequency energy storage elements, a four-quadrant operation is applied (i.e., bidirectional power flow) to maintain the waveform of the source current. The high value of the pulse frequency makes it possible to operate as an accurate and fast-reacting current generator capable of shaping the desired current waveform by the injection of a proper compensating current.

The system performs the function of an active filter optimizing the distortion of the source current. A power factor of one minimizes the distortion of the source current to $\approx 1 \%$ without the application of additional filters. Various values of the power factor can be obtained within the limits of the design criteria.

Compensation of the internal losses is executed by control logic integrated into the cascaded control loops.

The four-quadrant operation makes it possible to apply the discussed proposal to dc and ac motor drives.

\section{REFERENCES}

[1] F. C. Schwarz, "A time-domain analysis of the power factor for a rectifier filter system with over- and subcritical inductance," IEEE Trans. Ind. Electron. Control Instrumentation, vol. IECI-20, no. 2, pp. 61-68, May, 1973

[2] - , "Engineering information on an analog signal to discrete time interval converter," NASA CR-1344544, 1974, 175 p.
[3] M. Venturini, "A new sine wave in, sine wave out conversion technique eliminates reactive elements,"' in Proc. Powercon 7, San Diego, CA, March 1980, pp. E3-1-E3-15.

[4] F. C. Schwarz, and J. B. Klaassens, "A reversible smooth current source with momentary internal response for nondissipative control of multikilowatt de machines," IEEE Trans. Power Apparatus Syst., vol. PAS-100, no. 6, pp. 3008-3016, June 1981.

[5] J. B. Klaassens, "DC-to-AC series-resonant converter system with high internal frequency generating synthesized waveforms for multikilowatt power levels," IEEE Trans. Power Electron., vol. PE-1, no. 1, pp. 9-20, Jan. 1986

[6] E. Tironi, D. Zaninelli, and G. Ubezio, "Compensation of the reactive power by means of a current-controlled transistor inverter," 2nd European Conference on Power Electronics and Applications, Grenoble, France, Sept. 1987, pp. 1293-1299.

[7] G. van Schoor and J. D, van Wijk, "A study of a system of current fed converters as an active three phase filter," IEEE Power Electronics Specialists Conf., Blacksburg, VA, June 1987, pp. 482-490.

[8] E. Destobbeleer, G. Seguier, and A. Castelain, "AC-DC converter minimizing induced harmonics in industrial power systems," IEEE Trans. Power Electron., vol. PE-2, no. 4, pp. 320-327, Oct. 1987.

[9] J. B. Klaassens, and J. van Duivenbode, "Series-resonant energy conversion with multi-segment current waveforms for bipolar energy flow," IEEE Power Electronics Specialists Conf., Kyoto, Japan, April 1988, pp. 599-608.

[10] J. B. Klaassens, Moïze de Chateleux, W.L.F.H.A., and M. P. N. van Wesenbeeck, "Phase staggering control of a series-resonant dcdc converter with paralleled power modules," IEEE Trans. Power Electron., vol. PE-3, no. 2, pp. 164-173, April 1988.

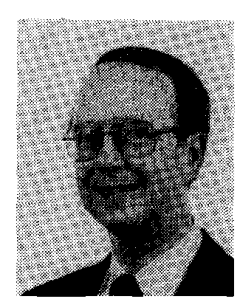

J. Ben Klaassens was born in Assen, The Netherlands in 1942. He received the B.S., M.S., and $\mathrm{Ph} . \mathrm{D}$. degrees in electrical engineering from the Delft University of Technology in The Netherlands.

He is currently Associate Professor at Delft University of Technology teaching graduate courses in the power electronics. His work has been concerned with inverter circuits, pulse-width modulation, and the control of electrical machinery. His research work and professional publications are in the area of converter systems with high internal pulse frequencies for submegawatt power levels employing thyristors, power transistors, and IGBT's. Dr. Klaassens has published a variety of papers on series-resonant converters for low- and high-power applications. He has designed and built prototypes of the early dc-dc to the recent ac-ac seriesresonant converters for a wide variety of applications, such as electric mo- 
tors and generators, communication power supplies, radar signal generators, arc welders, and space applications.

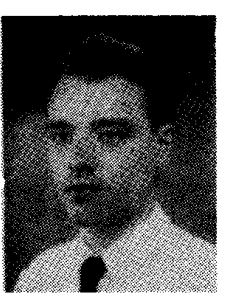

M. P. N. van Wesenbeeck received the B.S. de gree in electrical engineering and the M.S. in electrical engineering from the Delft University of Technology in The Netherlands in 1987 and 1989 , respectively. After graduation he joined the Power Electronics and Electrical Machines Group at Delft University of Technology.

$\mathrm{He}$ is currently involved in the research of medium-power soft-switching series-resonant software-controlled power converters as part of his Ph.D. study. His professional interests include electrical drive systems and power electronics.

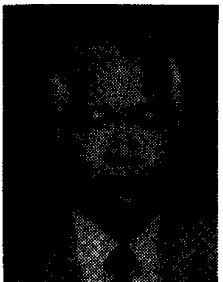

Hian K. Lauw received the MSEE and Ph.D. degrees from the Delft University of Technology, Delft, The Netherlands, in 1968 and 1976, respectively.

He was with the Department of Electrical Engineering of Delft University from 1968 till 1976 and joined the Department of Electrical and Computer Engineering at Oregon State University in 1978. He reduced his time commitment to his employment at Oregon State University in 1988 and assumed the position of Vice-President of Engineering at Electronic Power Conditioning, Inc. in 1988. Dr. Lauw's interest in research is in the field of electromechanical energy conversion and power electronics and, in particular, the application of the series-resonant converter technology as a controller for variable-speed drives and variablespeed generation. He has designed and built series-resonant converters at ratings ranging from $2-100 \mathrm{kVA}$. 